\title{
Interconnection, differentiation and bottlenecks in the Internet*
}

\author{
Emanuele Giovannetti ${ }^{\dagger}$ \\ University of Cambridge, \\ and University of Rome "La Sapienza".
}

\begin{abstract}
In the Internet information packets are routed through many vertically related hops, however these network hierarchies are not fixed. Two providers can be simultaneously vertically related, as supplier and retailer, in a routing process while being horizontally competing in another. We study pricing and interconnection decisions when ISPs become interconnected into a wider network. We find that interconnection lowers retail and access prices only when the downstream industry is poorly differentiated. The profitability of interconnection also depends on the differentiation of the retail sector. Interconnection is, however, universally agreed upon for an intermediate range of retail services differentiation values.
\end{abstract}

JEL Classification: L13, L86, L96,

Keywords: Access Pricing, Internet, Network Industries, Interconnection.

*Emanuele Giovannetti, DAE, Austin Robinson Building, Sidgwick Avenue, Cambridge, CB3 9DE, U.K. Tel. +44 1223 335274, Fax +44 1223 335299. E-mail: E.Giovannetti@econ.cam.ac.uk.

†I would like to thank: Richard Gibbens, Frank Kelly and David Newbery for useful advice and discussions at different stages of this paper. The usual disclaimer applies. 


\section{Introduction}

The brief history of the Internet ${ }^{1}$ had a major turning point in the 1995 with the end of the network financing by the National Science Foundation. Deregulation and opening to competition ${ }^{2}$ in the telecommunications sector and commercialization of the backbone services made Internet pricing a relevant economic problem to be analysed in an industry characterized by competing oligopolies and local bottlenecks.

When two final users are connected through the Internet, a network of networks, their communication exchanges are decomposed into information packets that can be routed through a multiplicity of networks. Often these information exchanges cross part of the network in North America, even if neither the source nor the recipient of the information exchange are located there. Indeed, more than half of intra-European and intra-Asian traffic passes through Network Access Points located in the United States.

The main feature of Internet Protocol (I.P.) based networks is that, albeit there is a different number of vertically related hops through which an information packet is routed from sender to receiver, these network hierarchies are not fixed. The vertical relation between two adjacent nodes can indeed be reversed for different information transfers. Even more interestingly, two providers owning different network nodes can be at the same time vertically related, as supplier and retailer, in a routing process while being horizontally competing for the routing of different traffic.

In this paper we study pricing and interconnection decisions where Internet Service Providers route traffic without being able to discriminate, or at least price discriminate, whether the information packets come from consumers' final demand or downstream providers' access demand.

We focus on the behavior of bottlenecks, local monopolies, when their original network becomes interconnected into a wider one. Interconnection is represented as a change in the pre-existing network architecture. Before interconnection we consider a traditional vertically ordered industry: where an upstream monopolist chooses an access price for the use of its bottleneck essential facility and a downstream differentiated duopoly sets retail prices to access the network edges. After interconnection a new dimension is added to the network and the former monopolist starts competing, as a retail service

\footnotetext{
${ }^{1}$ See for example Hobbes and Zakon [9].

${ }^{2}$ See, for example, Economides [4].
} 
provider, against its downstream retailers for the routing of through-traffic across the network.

The modelling of this, only partially ordered, hierarchy between adjacent nodes is used to capture the connection-less ${ }^{3}$ nature of the I.P. protocol.

The main questions we ask are: will interconnection affect the previous monopolistic pricing behavior of the local bottleneck? And if yes in which way? Will profits and/or retail and access prices increase or decrease due to interconnection and through-traffic? Will the outcome depend on the possibility of price discriminate between packets arising from different networks?

We find that the answers depend on the degree of product differentiation of the retail sector. This in turn reflects the trade off between consumers' preferences for variety in network access services and the transaction costs due to multiple connections. Preferences for variety are likely to be affected by the appearance of new access standards either in terms of speed, as with the ADSL (Asymmetric Digital Subscriber Line) ${ }^{4}$ or the VDSL (Very high rate Digital Subscriber Line $)^{5}$ connections, or of extended portability as with the WAP (Wireless Application Protocol) or UMTS (Universal Mobile Telecommunications Systems) protocols. Extending the compatibility between access to an I.P. network and existing devices such as mobile phones, copper or optical cables, television sets, or even wrist watches will indeed

\footnotetext{
${ }^{3}$ The route of an end to end information exchange in the Internet is recalculated at each step of the process, following statistical optimization algorithms.

4"ADSL is a new modem technology, converts existing twisted-pair telephone lines into access paths for multimedia and high speed data communications. ADSL transmits more than $6 \mathrm{Mbps}$ (optionally up to $8 \mathrm{Mbps}$ ) to a subscriber, and as much as $640 \mathrm{kbps}$ (optionally up to $1 \mathrm{Mbps)}$ more in both directions. Such rates expand existing access capacity by a factor of 50 or more without new cabling. ADSL can literally transform the existing public information network from one limited to voice, text and low resolution graphics to a powerful, ubiquitous system capable of bringing multimedia including full motion video, to everyone's home this century". (from www.adsl.com)

5 "In simple terms, VDSL transmits high speed data over short reaches of twisted-pair copper telephone lines, with a range of speeds depending upon actual line length. The maximum downstream rate under consideration is between 51 and $55 \mathrm{Mbps}$ over lines up to $1000 \mathrm{ft}$ (300 meters) in length. Downstream speeds as low as $13 \mathrm{Mbps}$ over lengths beyond $4000 \mathrm{ft}$ (1500 meters) are also in the picture. Upstream rates in early models will be asymmetric, just like ADSL, at speeds from 1.6 to $2.3 \mathrm{Mbps}$. Both data channels will be separated in frequency from bands used for POTS and ISDN, enabling service providers to overlay VDSL on existing services. At present the two high speed channels will also be separated in frequency. As needs arise for higher speed upstream channels or symmetric rates, VDSL systems may need to use echo cancellation." (www.adsl.com)
} 
offer more variety and an increased willingness to combine sources of access.

The more consumers consider the retail services, of different providers, as substitutes commodities the more competitive the retail industry becomes. When, on the contrary, new products or technologies increase the scope of I. P. network access modalities, the industry becomes more differentiated and less competitive.

We find that interconnection lowers retail and access prices, and therefore increases the local sending rates, only when the downstream industry is poorly differentiated.

The profitability of interconnection also depends on the degree of competitiveness of the retail sector. The original bottleneck prefers to interconnect, opening to through-traffic, only when there is enough differentiation. The incentives for interconnection in the originally downstream sector will instead depend on the new roles played by each provider after opening to through traffic. Interconnection is, however, universally agreed upon for an intermediate range of the parameter values describing the degree of differentiation in the retail sector.

We conclude by looking at the effects of price discrimination, the technological or regulatory possibility to charge different prices between packets depending on whether they arise from local or through-traffic. We find that with interconnection and opening to through-traffic, the original equilibrium prices are unchanged if the marginal routing costs are constant. This is not surprising since price discrimination across separate sources of traffic "dismantles" the interdependence of the Network architecture, typical of the Internet.

After a stylized description of the linkages between Internet users and a brief review of some related literature, the remainder of the paper is organized as follows: in section 2 we describe the model: consumers utility, demand for sending rates, equilibrium prices and profits and the effects of product differentiation for the network before interconnection. Section 3 contains the main results of the paper, in it we extend the original network architecture to introduce through-traffic and interconnection. We study the effects of this new network structure on retail and access prices, sending rates and incentives to interconnection. Finally section 4 contains the conclusions of the paper. All the proofs are contained in the Appendix. 


\section{$1.1 \quad$ Linkages}

The Internet, as a network of networks, has many major players ${ }^{6}$. In our model we provide a stylized representation of:

- Internet Service Providers (ISP's) providing access to the net.

- Network Access Points, NAPs and Internet Exchange Points, IXPs forming the physical interfaces between networks, and

- Internet Backbone Providers(IBPs) which carry data traffic, along long distance on fiber optic cables.

Each single network is connected along two dimensions with the rest of the net: by sharing the same $\mathrm{TCP} / \mathrm{IP}^{7}$ communication protocols and through physical linkages with NAPs.

In Figure 1, below, we provide a sketch of the possible linkages between two final users through the Internet. Consider ${ }^{8}$ an end user, A, he connects to a Point of Presence (POP) of its Internet Service Provider. If the information exchange is between $\mathrm{A}$ an other end user, $\mathrm{B}$, connected to a different ISP , then A's ISP needs to lease lines to transfer A's message from its POP to either a NAP or an Internet Exchange Point. If such point is shared by the sender's and the receiver's ISPs, then the path is decided, otherwise the IP protocol will evaluate the path, for each single information packets, to reach the B's ISP's Point of Presence.

This structure of direct or indirect connection between ISPs, NAPs and IXPs can be repeated many times depending on the actual distance, in the network topology, between the two users, A and B.

\footnotetext{
${ }^{6}$ It is important to keep in mind that overlap of these functions are often observed, also because of the recent trend in vertical integration, and mergers in the industry. For more details see Valletti and Cambrini [13].

${ }^{7}$ Transmission Control Protocol/ Internet Protocol, the communication protocol providing a common language allowing computers to exchange information.

${ }^{8}$ We only consider a stylized description of the linkages between users, abstracting from the technical aspects underlying them.
} 


\section{Linkages}

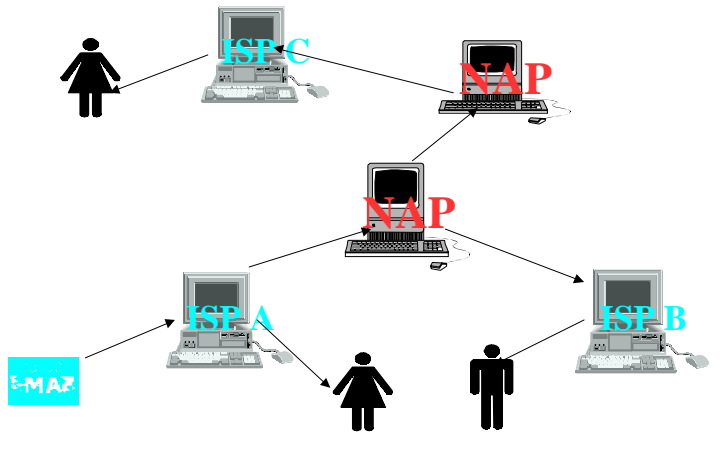

Figure 1

Long Distance connection is finally provided by backbone operators. The entire route is recalculated at each IXP-NAP, and the actual transmission process is carried by many different ISPs located at different hierarchical levels.

Figure 2 shows the physical interconnection, across the globe, of two commercial backbones the CWIX and Telstra.

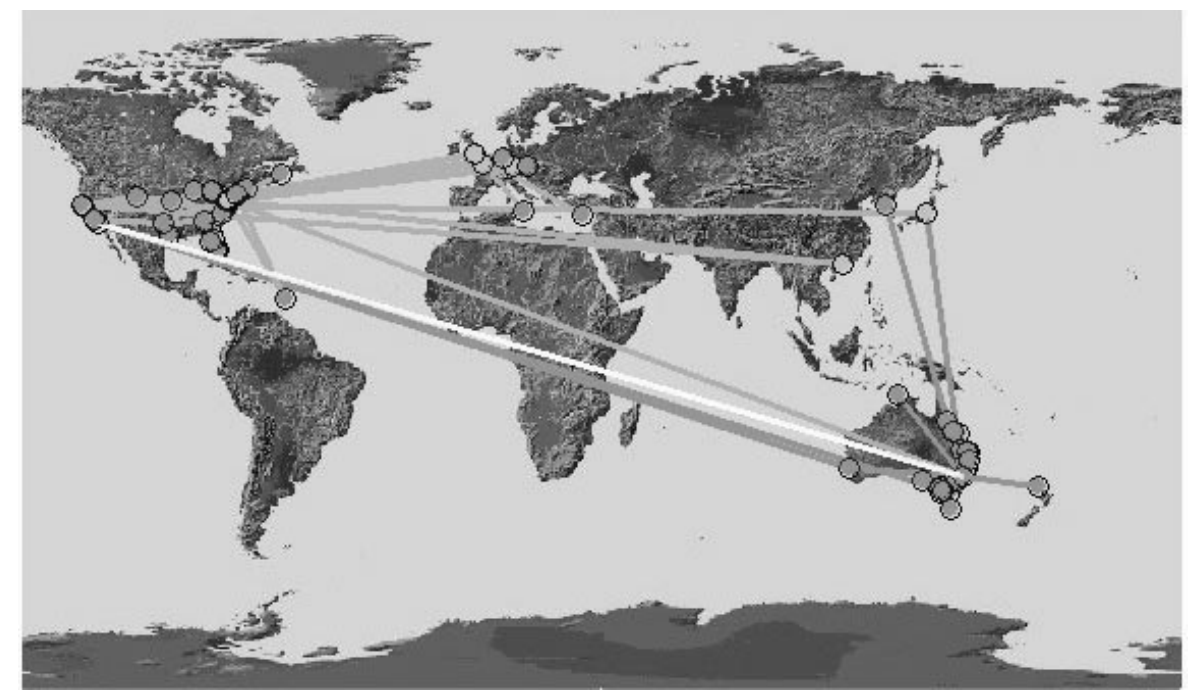

Figure 2 Telstra and CWIX networks. (Source www.mapnet.com) 


\section{$1.2 \quad$ Related literature}

The literature on Internet pricing has mainly focussed on congestion pricing ${ }^{9}$. One of the debated issues is whether prices should be charged only at the retail level, or if information packets should be priced at each hop along their path, following a motorway toll metaphor. Schenker, Clark, Estrin and Herzog, [12] suggested ${ }^{10}$ to use Edge Pricing, where the entire computation of the charges for the user is done at the access point: an ISP charges the users of its network and then makes a sequence of bilateral agreements with adjacent providers. In the following we also assume Edge Pricing, so that final users will only pay at the edges of the network a unit, per packet, price.

Strategic pricing problems in network industries have been analysed in details in the economic literature ${ }^{11}$, mostly for traditional telephony networks, where telephone companies direct and terminate calls to each other. In this setting a firm is located upstream when providing access for termination of external calls to its customers and downstream when demanding access for directing its customers calls outside its own network. These two-way networks models, see for example Armstrong [1] and Laffont, Rey and Tirole [10] are appropriate for telephone exchanges which take place along dedicated connections. In our paper we focus on a different network architecture aimed at capturing the connection-less features of I.P. networks. Instead of having two networks, each controlling an essential facility for the other (the access for its customers) we study bottleneck pricing, when the owner of the essential facility for one source of traffic also competes against its downstream retailers for the routing of through-traffic. Product differentiation remains however the crucial element for the conclusions derived in both our and the two-way networks models.

\footnotetext{
${ }^{9}$ For a collection of material on Internet congestion pricing see, for example, Richard Gibbens' web page [8] and for a seminal economic paper see MacKie-Mason and Varian [11].

${ }^{10}$ They criticized the possibility of using optimal pricing based on congestion costs. Charging in term of traffic has in facts the added complication that often connections involve bidirectional exchange of packets, as for example one downloads Web pages or uses File Transfer Protocol to get files from specific servers. Furthermore communication exchanges often involve the packet transfers across different networks, with different interconnection, access, peering and pricing agreements (for peering agreements see, for example, the Danish Internet Exchange framework [3])

${ }^{11}$ For an overview on the theory of access pricing and welfare considerations see Estache and Valletti [7]
} 
Our analysis of equilibrium pricing before interconnection follows Economides and Woroch ${ }^{12}[6]$. We extend their analysis, of a vertically ordered industry structure, to capture the effects of interconnection. Further research is required to compare their finding on divestures and merger incentives in our different network architecture.

\section{The Model: one continent}

We consider a network connecting two locations, $B_{1}$ and $B_{2}$, think about the two sides of a continent, connected by two alternatives routes. Each route linking the two locations passes through two local switches, (the four small boxes, in Figure 3), one at each end of the link.

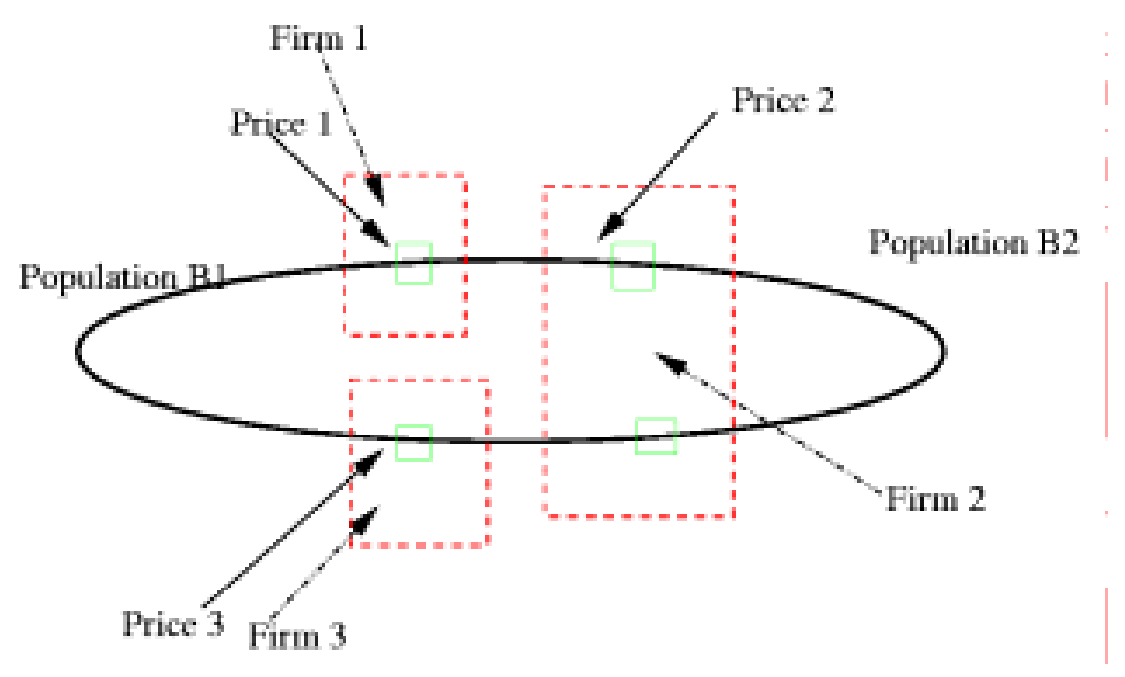

Figure 3

We assume that $B_{1}$ has a liberalized local switch market with two independent and competitive providers, say a cable company and the, previously monopolistic, local telephone company. $B_{2}$, on the contrary, has a single monopolistic local provider. The larger dashed boxes in Figure 3 show the ownership structure of the switches.

The total sending rate from location $\mathrm{B}_{1}$ to location $\mathrm{B}_{2}$ is given by

$$
x=x_{1}+x_{3}
$$

\footnotetext{
${ }^{12}$ For similar studies see also Armstrong, Doyle and Vickers [2] and Economides [5].
} 
where $x_{1}$ is the packet rate going through firm one and $x_{3}$ is the packet rate going through firm two.

\subsection{Sending rates utility}

In the following we assume that the preferences of a representative consumer are described by a quasilinear utility function:

$$
U=\left(\alpha\left(x_{1}+x_{3}\right)-\left(\frac{1}{2}\left(x_{1}^{2}+x_{3}^{2}\right)\right)-\gamma\left(x_{1} x_{3}\right)+m\right)
$$

where $x_{1}$ is the sending rate through provider one, and $x_{3}$ is the sending rate through provider three. The quasi linearity assumption implies a focus on price substitution effects ${ }^{13}$ : the impact on the demand for sending of changes in the providers relative prices.

The main interest for the following analysis lies in the terms composing the non linear part of the utility function:

$$
\left(\alpha\left(x_{1}+x_{3}\right)-\frac{1}{2}\left(x_{1}^{2}+x_{3}^{2}\right)-\gamma\left(x_{1} x_{3}\right)\right) .
$$

The first term, $\alpha\left(x_{1}+x_{3}\right)$, does not differentiate between accessing the network through provider one or provider three, what matters is the total sending rate, $x=\left(x_{1}+x_{3}\right)$, and the parameter $\alpha$ representing the size of the market.

The quadratic part of the utility function, second and third terms together:

$$
-\frac{1}{2}\left(x_{1}^{2}+x_{3}^{2}\right)-\gamma\left(x_{1} x_{3}\right)
$$

gives the concavity of this utility function ${ }^{14}$.

In particular the second term:

$$
-\frac{1}{2}\left(x_{1}^{2}+x_{3}^{2}\right)
$$

describes the consumers willingness of mixing different sources of access.

\footnotetext{
${ }^{13}$ The quasi linearity of the utility function also implies that we abstract from income effects due to retail price changes.

${ }^{14}$ The second partial derivatives with respect to the sending rates $x_{1}$ and $x_{3}$ are indeed always negative.
} 
This has an immediate interpretation: suppose we can access the network either via a cable television network, via a dial-up traditional telephone connection or via a UMTS mobile connection; this variety of access modes not only allows the use of the most convenient connection at each moment, but also allows multiple simultaneous connections.

Finally the third term:

$$
-\gamma\left(x_{1} x_{3}\right)
$$

accounts for the disutility derived from having two different providers. This disutility from variety expresses the opportunity costs for the time spent reading different contracts and bills, paying them separately, accessing two different support numbers for any query, learning different aspects of the connection, changing the configuration of your software if they are accessed from the same piece of hardware, and so on.

The parameter $\gamma$ which will be crucial in the following analysis, describes the trade off between the desire for variety and the effort to minimise transaction and opportunity costs. The more network access technologies are differentiated between providers, the lower $\gamma$ will be ${ }^{15}$.

When $\gamma$ tends to one there is no preference for variety: the transaction costs associated with having two different providers, or two different ways of accessing the web, exactly offset the benefits derived from variety ${ }^{16}$.

\subsection{Demand for sending rates}

In the following we assume that the price of an information exchange over the I.P. network, between location $B_{1}$ and $B_{2}$, is given by the call rate, $x_{i}$, (the packet sending rate) times the price, $p_{i}$, imposed by either of the local switches in $B_{1}$.

\footnotetext{
${ }^{15}$ In the following we assume that $\gamma$ is always positive but less than one; this implies that the second term of the utility function, inducing a preference for variety is stronger than the third term, the costs of having two different providers.

${ }^{16}$ The role played by $\gamma$ on consumers preferences can be read by drawing indifference curves. If a consumer is completely indifferent between the use of the two providers, when $\gamma$ equals one, the resulting indifference curve is a straight line. In this case the two sending rates are perfect substitutes commodities.

If, on the contrary, mixing is preferred, or $\gamma$ is below one, the indifference curve will be concave towards the origin.
} 
Market demands for access are derived maximising the quasi linear utility function discussed in the previous section subject to a Budget constraint.

The inverse demand functions are:

$$
\begin{aligned}
& p_{3}=\alpha-x_{3}-\gamma x_{1} \\
& p_{1}=\alpha-x_{1}-\gamma x_{3}
\end{aligned}
$$

and the direct ones:

$$
\begin{aligned}
& x_{3}=a-b p_{3}+c p_{1} \\
& x_{1}=a-b p_{1}+c p_{3}
\end{aligned}
$$

where

$$
a=\frac{\alpha(1-\gamma)}{1-\gamma^{2}}, b=\frac{1}{1-\gamma^{2}}, c=\frac{\gamma}{1-\gamma^{2}}
$$

The direct demand functions provide another interpretation for the parameter $\gamma$. In these functions the parameter $b$, represents the own price effect, or how a variation of a firm's own price will affect its market demand, and the term $c$, the cross price effect, expresses the effect on a firm's demand of a variation of its competitor's price. The parameter $\gamma$ given by the ratio of these two effects, $\gamma=\frac{c}{b}$, translates into a powerful indicator of the degree of differentiation and competition in the retail network access industry. When $\gamma$ equals zero, there is no competition effect and each firm has a safe market niche, when, on the contrary, $\gamma$ tends to one the industry becomes perfectly competitive.

\subsection{Timing of the game}

The timing of the price setting process is extremely relevant.

In the simple network analysed in this section there is a clear vertical distinction between the retail sector, firm one and firm three, and the upstream monopoly, firm two. It is therefore reasonable to assume that the timing of the price setting decision is sequential: first firm two, the local bottleneck, sets its access charge, and then firm one and three, knowing the access price they have to pay to firm two, simultaneously choose their retail prices. 
Finally we assume that the variable costs of routing a rate $x_{i}$ is zero at each network note, and that the fixed cost is zero, or sunk. Firm one and three need to pay an access price to the bottleneck firm two equal to $p_{2}$.

\subsection{Prices, profits and sending rates}

We are now ready to analyse the properties derived from the Nash Equilibria of a game where the three firms, of Figure 3, compete in prices charged for the routing of the packets through their own switches. In the next proposition we look at the equilibrium prices, profits and sending rates of this game.

\section{Proposition 1}

i) The retail equilibrium prices are given by:

$$
p_{1}=p_{3}=\frac{1}{2} \alpha \frac{2 \gamma-3}{\gamma-2}
$$

and the access price set by the monopolist is given by:

$$
p_{2}=\frac{1}{2} \alpha \text {. }
$$

ii) The equilibrium sending rates are:

$$
x_{1}=x_{3}=-\frac{1}{2} \frac{\alpha}{(\gamma+1)(\gamma-2)}
$$

and total traffic:

$$
X=-\frac{\alpha}{(\gamma+1)(\gamma-2)}
$$

iii) Downstream profits are given by:

$$
\pi_{1}=\pi_{3}=-\frac{1}{4}(-1+\gamma) \frac{\alpha^{2}}{(-2+\gamma)^{2}(\gamma+1)}
$$

while the bottleneck ones are: 


$$
\pi_{2}=\frac{1}{2} \alpha\left(-\frac{\alpha}{(\gamma+1)(\gamma-2)}\right)
$$

The proof is in the Appendix.

Notice that with the assumption of, $\gamma<1$, all equilibrium prices are positive. This condition, discussed before, is that the own price effect is stronger than the cross price effect.

\subsection{The role of network access services differentiation}

We anticipated that the parameter $\gamma$ would have relevant effects on the features of the industry. The next corollaries study these effects on equilibrium prices, profits and sending rates.

\section{Corollary 1}

i) Retail prices, $p_{1}$ and $p_{3}$, are decreasing in $\gamma$, while the access price $p_{2}$ is constant. When $\gamma$ tends to one, retail prices converge to the retail firms' costs: the access price, $p_{2}$, charged by the bottleneck.

ii) Retail profits are decreasing in $\gamma$ and their limit for $\gamma \rightarrow 1$ is zero $^{17}$

\section{Corollary 2}

i) Total traffic, $X$, is first decreasing and then increasing in $\gamma$.

ii) The monopolist profits follow the same pattern of the total traffic.

\section{Discussion}

The U shaped relation between total traffic and $\gamma$, shows the two different roles played by this parameter. The positive effect of $\gamma$ on total traffic is due to the inverse relation between $\gamma$ and the retail prices, because of the

\footnotetext{
${ }^{17}$ When retail prices equal access prices we obtain the Bertrand result of zero profits for the retail sector. This is a special case appearing when the cross price effect tends to the own price effect, or when the willingness to mix disappears because of relevant transaction costs, or a lack of appealing variety.
} 
smaller mark up ${ }^{18}$ imposed by the retail sector when network access services are not very differentiated. The negative effect shows the role of product differentiation on final demand: indeed the price insensitive component of the final demand function, $\frac{\alpha(1-\gamma)}{1-\gamma^{2}}$, which is decreasing in $\gamma$, expresses the positive effect of product differentiation on market expansion for given retail prices. For $\gamma<\frac{1}{2}$ the negative effect on market size of a reduction in product differentiation dominates the positive effect of a reduction in retail prices. For $\gamma \geq \frac{1}{2}$ the price effect becomes stronger. As a result total traffic is maximised either when the technology of access to the network are highly differentiated, or when they are homogenous. The plot of total traffic, as a function of the parameter $\gamma$, below shows this clearly ${ }^{19}$ :

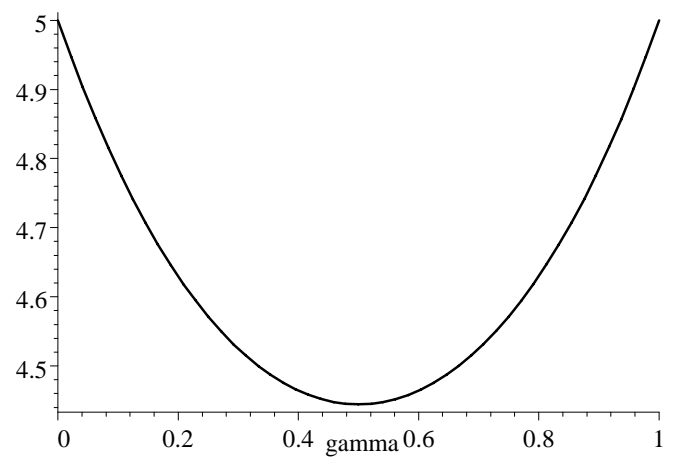

Figure 4. Total traffic as a function of gamma

\section{Interconnected Networks: three continents}

In this section we have the main results of the paper. We consider the effects of opening to through traffic as the original network becomes interconnected with external traffic flows.

Suppose that the communication exchanges between two more locations, $A_{1}$ and $A_{2}$, for example Japan and Europe, are routed through the original network studied in the previous section.

\footnotetext{
${ }^{18}$ This is a second mark-up, after the one imposed by the monopolist.

${ }^{19}$ The plot of the monopoly profits is just a rescaled version of this one since the access price does not change with gamma.
} 


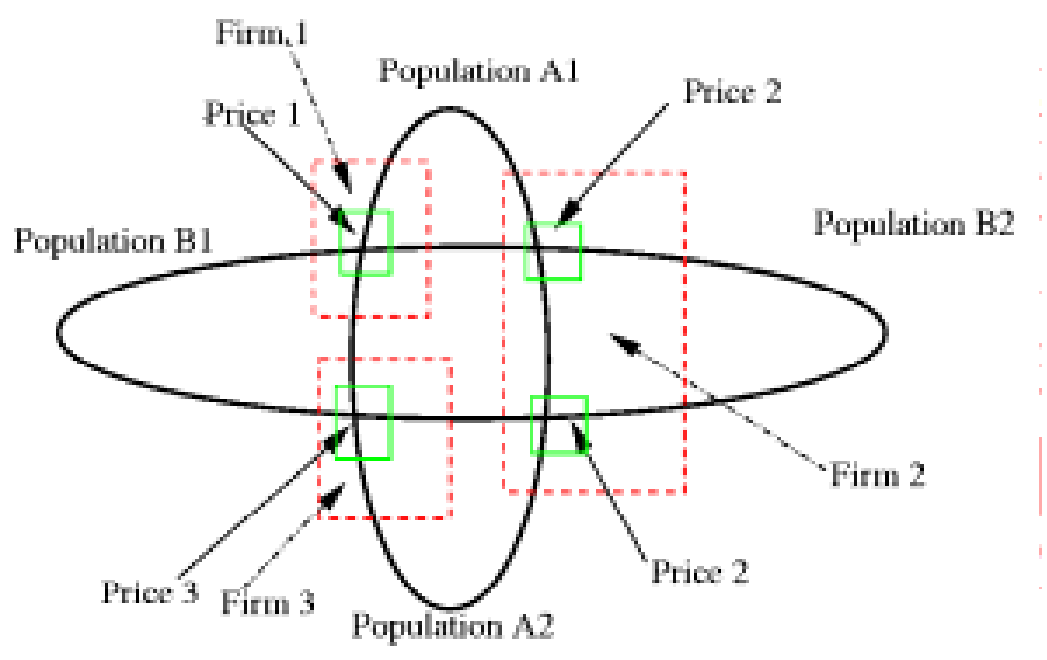

Figure 5

When $A_{1}$ wants to call $A_{2}$ they can either choose to pass through the two switches on the $B_{1}$ side, owned separately by firms 1 and 3 , or through the two switches on the $B_{2}$ side, owned by the monopolist, firm 2. See Fig 5 .

We assume that firm 2 , having monopoly rights on $B_{2}$ but competing against firm one for the routing of the through-traffic, is not able to price discriminate between packets originating either in $A_{1}$ or in $B_{1}$.

Through-traffic is modeled as additional final demand for firms one and two. These new demand functions are expressed by:

$$
x_{1}^{A}=\frac{\alpha(1-\gamma)}{1-\gamma^{2}}-\frac{1}{1-\gamma^{2}} p_{1}+\frac{\gamma}{1-\gamma^{2}} p_{2}
$$

and

$$
x_{2}^{A}=\frac{\alpha(1-\gamma)}{1-\gamma^{2}}-\frac{1}{1-\gamma^{2}} p_{2}+\frac{\gamma}{1-\gamma^{2}} p_{1}
$$

\subsection{Timing of the game}

Now $p_{2}$ is both an access price, charged to firms one and three, on the $B$ original traffic, and a final price for the through-traffic coming from $A ; p_{1}$ is 
a final price for both locations $A$ and $B$, while $p_{3}$, is a retail price for $B$ and an access price charged to firm 1 for the through-traffic originating in $A$.

Interconnection changes the topology of the network and therefore firms set now, without the possibility to discriminate, access and retail prices. We consider, therefore, that by allowing through-traffic $p_{1}, p_{2}$, and $p_{3}$ are all chosen simultaneously. The opening to through-traffic changes the timing of price setting decisions because it decomposes the original vertical relations into an interconnected network, with only partially ordered hierarchies.

\subsection{Retail and access prices after interconnection}

In the following proposition we derive the new equilibrium prices for the interconnected networks.

\section{Proposition 2}

Equilibrium prices with through-traffic are given by

$$
\begin{aligned}
& p_{1}=(-1+\gamma) \alpha \frac{3 \gamma+7}{3 \gamma^{2}+3 \gamma-8} \\
& p_{2}=(-1+\gamma) \alpha \frac{3 \gamma+5}{3 \gamma^{2}+3 \gamma-8} \\
& p_{3}=(-1+\gamma) \alpha \frac{3 \gamma+7}{3 \gamma^{2}+3 \gamma-8}
\end{aligned}
$$

The Proof is in the Appendix.

These prices are positive given the assumption that $\gamma<1$, or again if the own price effect is stronger than the cross price effect.

\section{Corollary 3}

At these prices equilibrium sending rates are positive and are given by: 


$$
\begin{aligned}
x_{1}^{B} & =-\frac{\alpha}{3 \gamma^{2}+3 \gamma-8} \\
x_{3}^{B} & =-\frac{\alpha}{3 \gamma^{2}+3 \gamma-8} \\
x_{1}^{A} & =\alpha \frac{-1+\gamma}{\left(3 \gamma^{2}+3 \gamma-8\right)(\gamma+1)} \\
x_{2}^{A} & =-(\gamma+3) \frac{\alpha}{\left(3 \gamma^{2}+3 \gamma-8\right)(\gamma+1)}
\end{aligned}
$$

\subsection{The effects of interconnection on prices and send- ing rates}

The first question we want to ask is what is the effect of interconnection on the equilibrium prices? Do retail and access prices increase or decrease because of interconnection? We will see that the answer will again reflect the retail market competition conditions, determined by the consumers willingness to mix sending rates.

In the next proposition we find that retail prices decrease after interconnection only when the retail access services are not very differentiated.

\section{Proposition 3}

i) For all values of $\gamma$, such that $\gamma \lesssim 0.70$, the retail prices, $p_{1}$ and $p_{3}$, are lower, and the sending rates higher, before interconnection, while for $\gamma \gtrsim 0.70, p_{1}$ and $p_{3}$ are lower, and the sending rates higher, after interconnection.

ii) For all values of $\gamma$, such that $\gamma \lesssim 0.67$ the bottleneck price, $p_{2}$, is lower before interconnection while for $\gamma \gtrsim 0.67, p_{2}$ is lower after interconnection.

This is easily seen, indeed the retail price differential ${ }^{20}$ for the downstream sector for any value of $\alpha$, is positive for $\gamma \lesssim 0.70$ and negative thereafter:

\footnotetext{
${ }^{20}$ Equilibrium prices after interconnection minus equilibrium prices before interconnection: $\Delta p_{1}=\Delta p_{3}=-\frac{1}{2} \alpha \frac{\gamma^{2}+5 \gamma-4}{\left(3 \gamma^{2}+3 \gamma-8\right)(\gamma-2)}$.
} 


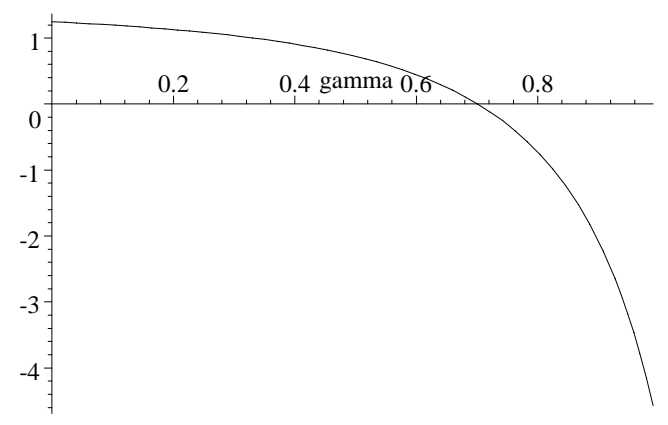

Figure 6. The downstream price differential

Only with strong competition in the retail sector due to poorly differentiated network access modalities, interconnection lowers prices.

The first effect of the new equilibrium retail prices, is a change in the total sending rate at the original location, from $B_{1}$ to $B_{2}$, this is of course positive when interconnection lowers retail prices and negative when on the contrary prices after interconnection are higher ${ }^{21}$.

The global traffic level routed through the network now includes, of course, the extra through-traffic. Changes in total traffic are given by the changes in the sending rate at the original location plus the additional through-traffic and are always positive ${ }^{22}$ :

${ }^{21}$ The quantitative effect of interconnection on the original sending rate is given by:

$$
\Delta X^{B}=\frac{1}{\gamma+1} \alpha \frac{\gamma^{2}+5 \gamma-4}{\left(3 \gamma^{2}+3 \gamma-8\right)(\gamma-2)} .
$$

${ }^{22}$ These are given by:

$$
\Delta X^{B}+X^{A}=\alpha \frac{\gamma^{2}+\gamma+4}{(\gamma+1)\left(3 \gamma^{2}+3 \gamma-8\right)(\gamma-2)}
$$




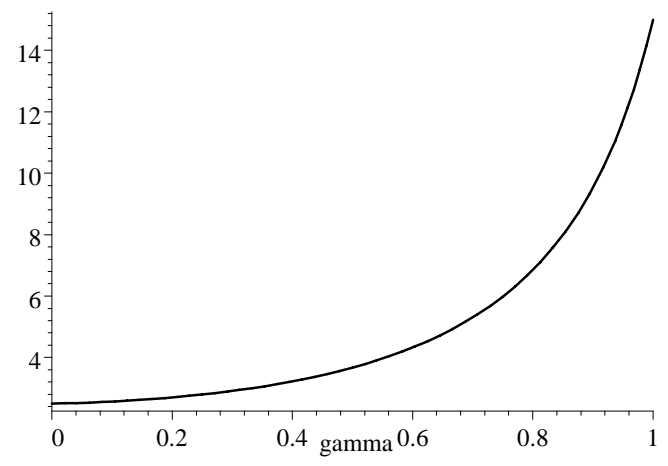

Figure 7. Changes in total traffic

Finally the bottleneck price differential expresses the effect of network interconnection on the original access price. The new price of the bottleneck remains indeed an access price for the retail sector of the original location, though now it also plays the role of a retail price for the through-traffic. For a differentiated retail industry, the access price is now higher after interconnection.

Only a competitive retail industries, with poorly differentiated retail services, implies a reduced bottleneck price after interconnection. The change in the access price in relation to $\gamma$ is described in the following picture $^{23}$ :

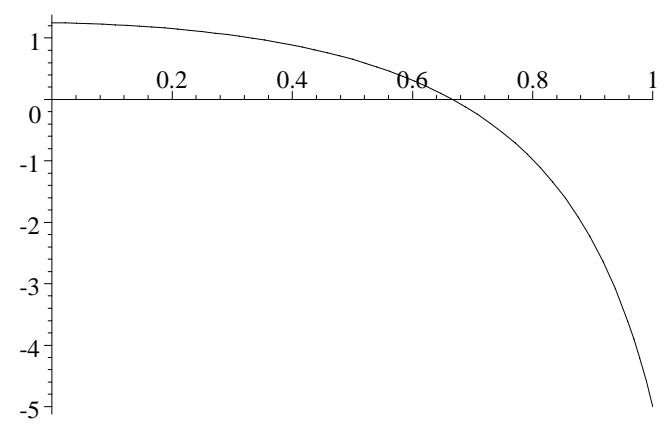

Figure 8. Access price differential.

\footnotetext{
${ }^{23}$ The analytical expression of the change in the access price, $p_{2}$, due to interconnection, is given by: $\Delta p_{2}=\frac{1}{2} \alpha \frac{\gamma-2+3 \gamma^{2}}{3 \gamma^{2}+3 \gamma-8}$.
} 


\subsection{Incentives to interconnect}

We are now ready to study the effects of through-traffic on providers' profits. Again, we will see that the role of the retail market competition, induced by the consumers willingness to mix service providers, is essential in deciding whether or not interconnection is profitable.

\section{Proposition 4}

Equilibrium profits with through-traffic are given by:

$$
\begin{aligned}
& \pi_{1}=-2(-1+\gamma) \frac{\alpha^{2}}{\left(3 \gamma^{2}+3 \gamma-8\right)^{2}} \\
& \pi_{2}=-\alpha^{2}(3 \gamma+5) \frac{3 \gamma^{2}+2 \gamma-5}{(\gamma+1)\left(3 \gamma^{2}+3 \gamma-8\right)^{2}} \\
& \pi_{3}=\alpha^{2} \frac{3 \gamma^{3}-\gamma^{2}-11 \gamma+9}{(\gamma+1)\left(3 \gamma^{2}+3 \gamma-8\right)^{2}}
\end{aligned}
$$

This is easily seen by substituting the equilibrium prices into the demand and profit functions.

The following corollary analyses the retail market conditions required for interconnection to be profitable for the three firms:

\section{Corollary 4}

i) For all values of $\gamma$, such that $\gamma \lesssim 0.62$ firm one prefers not to interconnect while for $\gamma \gtrsim 0.62$ its profits are higher after interconnection.

ii) For all values of $\gamma$, such that $\gamma \lesssim 0.95$ firm two prefers to interconnect while for $\gamma \gtrsim 0.95$ its profits are higher before interconnection.

iii) Firm three always prefers to interconnect.

This is easily seen ${ }^{24}$ in Figure 9 below: the solid plot shows the incentives to interconnect, by opening to through-traffic, as a function of $\gamma$,for firm one, the dashed plot shows it for firm two and the dotted plot for firm three:

\footnotetext{
${ }^{24}$ The profit differential for firm one is now given by:
} 


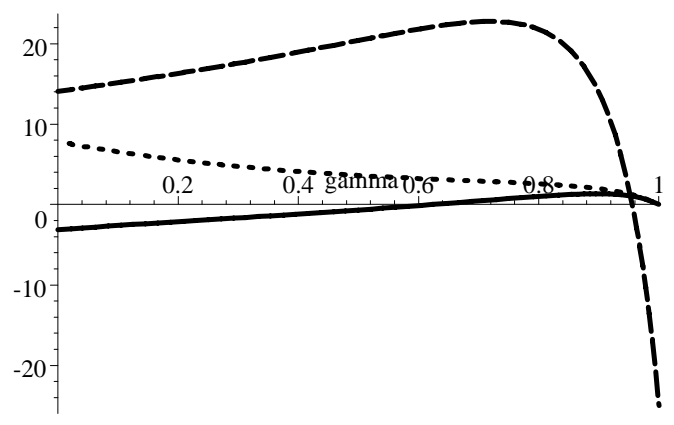

Figure 9. Incentives to interconnect.

If interconnection is decided on a triple coincidence of wants, it is clear that firm one is going to be the vetoing party for lower values of gamma, when the access services are highly differentiated. Indeed in the new network topology, firm one is the only one providing only retail services, while firm two and three also provide indirect access through their switches. Interconnection clearly has different effects depending on the role played within a network. Product differentiation, a low $\gamma$, affects negatively the profitability of interconnection for the retail only provider.

It is now interesting to see how the total industry profit differential changes with the degree of network access services differentiation. The total industry profits differential ${ }^{25}$ is plotted in Figure 10 below:

$$
\Delta \pi_{1}=\frac{1}{4}(-1+\gamma) \alpha^{2} \frac{10 \gamma^{3}-15 \gamma^{2}+32+9 \gamma^{4}-48 \gamma}{\left(3 \gamma^{2}+3 \gamma-8\right)^{2}(\gamma-2)^{2}(\gamma+1)}
$$

for firm two is:

$$
\Delta \pi_{2}=-\frac{1}{2} \alpha^{2} \frac{9 \gamma^{4}-12 \gamma^{3}-55 \gamma^{2}+18 \gamma+36}{(\gamma+1)\left(3 \gamma^{2}+3 \gamma-8\right)^{2}(\gamma-2)}
$$

and for firm three is:

$$
\Delta \pi_{3}=\frac{1}{4} \alpha^{2} \frac{21 \gamma^{5}-43 \gamma^{4}-37 \gamma^{3}+187 \gamma^{2}-208 \gamma+80}{\left(3 \gamma^{2}+3 \gamma-8\right)^{2}(\gamma-2)^{2}(\gamma+1)} .
$$

25

$$
\Delta \Pi=\frac{1}{2} \alpha^{2} \frac{-51 \gamma^{2}+96+9 \gamma^{4}-64 \gamma+6 \gamma^{5}}{\left(3 \gamma^{2}+3 \gamma-8\right)^{2}(\gamma-2)^{2}(\gamma+1)}
$$




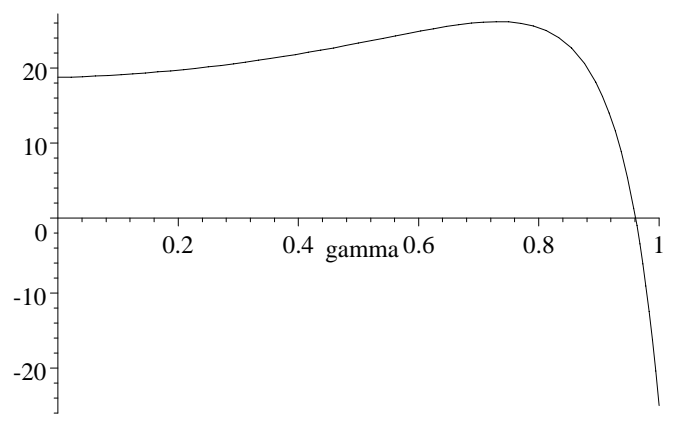

Figure 10.Total Industry profit differential.

Industry profits are higher after interconnection if $\gamma \lesssim 0.96$, and lower thereafter. This suggests that a redistribution of profits to the retail only provider can be used to implement interconnection when there is a high degree of differentiation.

\subsection{Discriminating packets originating at different lo- cations}

We conclude this study introducing the possibility of price discrimination between calls originating at different locations. The next proposition analyses the effects on the equilibrium prices, of a technological or regulatory change allowing each firm to charge different prices for routing calls depending on their original location.

\section{Proposition 5}

If Internet Service Providers can price discriminate among packets of different origins, $A$ or $B$, the integration of the original network into a wider one maintains equilibrium switch prices unchanged at the original location $B$ if there are no capacity constraints and marginal routing costs are constant.

This is obvious because the two pricing problems, how to price traffic originating in $B$ and through-traffic from $A$, are independent, given the assumption of constant marginal routing costs. Given this independence pricing at location $B$ is the same as before interconnection. 
The results of the previous sections describe what happens when one firm with local market power, a local bottleneck, needs to maintain the same price across a second market where it does not have such power. The relevance of such situation appears when a former local monopoly becomes integrated into a wider international network, and technological or regulatory factors do not allow price discrimination across markets. In our case the incentives to open to through-traffic are linked to the degree of differentiation of the network access services, as perceived in the users utility function. This in facts determines the equilibrium results by defining the degree of strategic complementarity in the strategic variables used, uniform prices across interconnected markets.

\section{Conclusions}

In this paper we found that network integration, without price discrimination, does affect equilibrium prices and profits of the providers given the described ownership structure and pricing scheme.

Interconnection lowers retail prices, and therefore increases the local sending rate, only when the cross price effect is strong enough, or when the downstream industry is competitive. We analysed the connection between consumers preferences and competitiveness of the industry. We found that the more consumer appreciate sending rates through different networks, for example because of different access technologies, the less competitive the industry is. If, on the contrary, consumers are indifferent about the possible ways of accessing the I.P. network the industry will become more competitive, and less profitable. We claimed that these preferences are shaped by the appearance of new network access and portability technologies.

The profitability of interconnection and opening to through-traffic also depend on the degree of competitiveness of the retail sector.

The original bottleneck, firm two, prefers to interconnect in less competitive settings.

In the downstream sector the provider setting the retail price also for the through-traffic, firm one, prefers to interconnect for high values of the cross price effect, while the provider setting the access price for the new through-traffic, firm three, always prefers interconnection.

With the possibility of price discriminate and if there are no capacity constraints and marginal routing costs are constant interconnection looses its 
interest since it does not alter the original equilibrium. With price discrimination the new physical linkages allowing interconnection and through-traffic, do not provide economic linkages across markets and the original identities remain unchanged.

\section{Appendix}

\section{Proof of Proposition 1}

To solve a two period game, where in the first period the upstream monopolist sets the access price and in the second period the downstream competing firms choose their retail prices, we start by analyzing the second period interaction. We solve for a Nash Equilibrium of the final stage game where firms one and three set their prices as a function of the known access price, $p_{2}$, set by firm 2 .

The profit functions in the downstream market are obviously given by the sending rates times the difference between the final price charged to the users, $p_{1}$, or $p_{3}$, minus the access price set by the bottleneck: $p_{2}$.

$$
\begin{aligned}
& \pi_{1}=\left(\frac{\alpha(1-\gamma)}{1-\gamma^{2}}-\frac{1}{1-\gamma^{2}} p_{1}+\frac{\gamma}{1-\gamma^{2}} p_{3}\right)\left(p_{1}-p_{2}\right) \\
& \pi_{3}=\left(\frac{\alpha(1-\gamma)}{1-\gamma^{2}}-\frac{1}{1-\gamma^{2}} p_{3}+\frac{\gamma}{1-\gamma^{2}} p_{1}\right)\left(p_{3}-p_{2}\right)
\end{aligned}
$$

From the first order conditions we obtain the system of best replies function for the second period of the game:

$$
\begin{aligned}
& p_{1}=\frac{1}{2} p_{3} \gamma+\frac{1}{2} p_{2}+\frac{1}{2} \alpha-\frac{1}{2} \alpha \gamma \\
& p_{3}=\frac{1}{2} p_{1} \gamma+\frac{1}{2} p_{2}+\frac{1}{2} \alpha-\frac{1}{2} \alpha \gamma
\end{aligned}
$$

which is solved by:

$$
\begin{aligned}
& p_{1}=-\frac{-\alpha \gamma+\alpha+p_{2}}{\gamma-2} \\
& p_{3}=-\frac{-\alpha \gamma+\alpha+p_{2}}{\gamma-2}
\end{aligned}
$$


In the first period of the game the bottleneck sets its access price $p_{2}$ to maximise its own profits by knowing how the downstream competitors will react to its own choice, knowing their second period reaction functions. Final demand for access to the bottleneck is given by the sum of the two sending rates, now a function of $p_{2}$.

Upstream profits are given by:

$$
\pi_{2}=p_{2}\left(x_{1}+x_{3}\right)=2 p_{2}\left(\frac{-\alpha+p_{2}}{(\gamma-2)(\gamma+1)}\right)
$$

maximised by the access price:

$$
p_{2}=\frac{\alpha}{2}
$$

Substituting (25)into(23) we obtain:

$$
\begin{aligned}
& p_{1}=\frac{1}{2} \alpha \frac{2 \gamma-3}{\gamma-2} \\
& p_{3}=\frac{1}{2} \alpha \frac{2 \gamma-3}{\gamma-2}
\end{aligned}
$$

\section{Proof of Proposition 2}

Opening to through traffic implies new demand functions for the three firms.

Demand for firm 1 is now given by:

$$
\begin{aligned}
x_{1}= & \left(\frac{\alpha(1-\gamma)}{1-\gamma^{2}}-\frac{1}{1-\gamma^{2}} p_{1}+\frac{\gamma}{1-\gamma^{2}} p_{3}\right)+ \\
& +\left(\frac{\alpha(1-\gamma)}{1-\gamma^{2}}-\frac{1}{1-\gamma^{2}} p_{1}+\frac{\gamma}{1-\gamma^{2}} p_{2}\right)
\end{aligned}
$$

and its profits are given by:

$$
\begin{aligned}
\pi_{1}= & \left(\frac{\alpha(1-\gamma)}{1-\gamma^{2}}-\frac{1}{1-\gamma^{2}} p_{1}+\frac{\gamma}{1-\gamma^{2}} p_{3}\right)\left(p_{1}-p_{2}\right)+ \\
& +\left(\frac{\alpha(1-\gamma)}{1-\gamma^{2}}-\frac{1}{1-\gamma^{2}} p_{1}+\frac{\gamma}{1-\gamma^{2}} p_{2}\right)\left(p_{1}-p_{3}\right)
\end{aligned}
$$


Notice that now firm one pays an access price, $p_{2}$, to firm two for the traffic originating at $B$, and an access price, $p_{3}$, for the traffic originating in $A$, while charging a unique price, $p_{1}$, to all its final demand.

Demand for firm 2 is given by

$$
\begin{aligned}
x_{2}= & \left(\frac{\alpha(1-\gamma)}{1-\gamma^{2}}-\frac{1}{1-\gamma^{2}} p_{1}+\frac{\gamma}{1-\gamma^{2}} p_{3}\right)+ \\
& \left(\frac{\alpha(1-\gamma)}{1-\gamma^{2}}-\frac{1}{1-\gamma^{2}} p_{3}+\frac{\gamma}{1-\gamma^{2}} p_{1}\right)+ \\
+ & \left(\frac{\alpha(1-\gamma)}{1-\gamma^{2}}-\frac{1}{1-\gamma^{2}} p_{2}+\frac{\gamma}{1-\gamma^{2}} p_{1}\right)
\end{aligned}
$$

where through-traffic is added to the previous demand for access.

Its profits are given by:

$$
\pi_{2}=p_{2}\left(-\frac{3 \alpha-3 \alpha \gamma-p_{1}+\gamma p_{3}-p_{3}+2 \gamma p_{1}-p_{2}}{-1+\gamma^{2}}\right)
$$

In this case firm two charges an access price only on the demand coming from external providers, firms one and three.

Finally demand for firm 3 is given by

$$
\begin{aligned}
x_{3}= & \left(\frac{\alpha(1-\gamma)}{1-\gamma^{2}}-\frac{1}{1-\gamma^{2}} p_{3}+\frac{\gamma}{1-\gamma^{2}} p_{1}\right)+ \\
& \left(\frac{\alpha(1-\gamma)}{1-\gamma^{2}}-\frac{1}{1-\gamma^{2}} p_{1}+\frac{\gamma}{1-\gamma^{2}} p_{2}\right)
\end{aligned}
$$

the final demand from $B$, and the demand for access arising from the routing of firm's one through-traffic. Firm's three profits are given by:

$$
\begin{aligned}
\pi_{3}= & \left(\frac{\alpha(1-\gamma)}{1-\gamma^{2}}-\frac{1}{1-\gamma^{2}} p_{3}+\frac{\gamma}{1-\gamma^{2}} p_{1}\right)\left(p_{3}-p_{2}\right)+ \\
& +\left(\frac{\alpha(1-\gamma)}{1-\gamma^{2}}-\frac{1}{1-\gamma^{2}} p_{1}+\frac{\gamma}{1-\gamma^{2}} p_{2}\right) p_{3}
\end{aligned}
$$

To construct best reply functions we need to consider the possibility that a firm sets prices "closing" one market. This is important because firms now 
play with one price on two different markets and we need to verify if corner solutions are best replies against other firms internal ones. By setting up Lagrangians for the three firms we find that the internal solutions form an equilibrium for the game.

Firm one Imposing the non negativity constraints for the two final demands routed by firm one, we have the Lagrangian function:

$$
\begin{aligned}
L_{1}= & \left(\frac{\alpha(1-\gamma)}{1-\gamma^{2}}-\frac{1}{1-\gamma^{2}} p_{1}+\frac{\gamma}{1-\gamma^{2}} p_{3}\right)\left(p_{1}-p_{2}\right)+ \\
& +\left(\frac{\alpha(1-\gamma)}{1-\gamma^{2}}-\frac{1}{1-\gamma^{2}} p_{1}+\frac{\gamma}{1-\gamma^{2}} p_{2}\right)\left(p_{1}-p_{3}\right)+ \\
& \mu_{1}\left(\frac{\alpha(1-\gamma)}{1-\gamma^{2}}-\frac{1}{1-\gamma^{2}} p_{1}+\frac{\gamma}{1-\gamma^{2}} p_{3}\right)+ \\
& \mu_{2}\left(\frac{\alpha(1-\gamma)}{1-\gamma^{2}}-\frac{1}{1-\gamma^{2}} p_{1}+\frac{\gamma}{1-\gamma^{2}} p_{2}\right)
\end{aligned}
$$

From the first order conditions we obtain:

$$
p_{1}=\frac{1}{4} \gamma p_{3}+\frac{1}{4} p_{2}-\frac{1}{2} \gamma \alpha+\frac{1}{2} \alpha+\frac{1}{4} \gamma p_{2}+\frac{1}{4} p_{3}-\frac{1}{4} \mu_{1}-\frac{1}{4} \mu_{2}
$$

First we consider the case when demand for firm one on the B market is zero:

$$
\left(\frac{\alpha(1-\gamma)}{1-\gamma^{2}}-\frac{1}{1-\gamma^{2}} p_{1}+\frac{\gamma}{1-\gamma^{2}} p_{3}\right)=0
$$

and

$$
\left(\frac{\alpha(1-\gamma)}{1-\gamma^{2}}-\frac{1}{1-\gamma^{2}} p_{1}+\frac{\gamma}{1-\gamma^{2}} p_{2}\right)>0
$$

in this case the first multiplier, $\mu_{1}$, must be greater than zero, while the second constraint is not biting and therefore we have: $\mu_{2}=0$. The first order condition (34)becomes:

$$
p_{1}=\frac{1}{4} \gamma p_{3}+\frac{1}{4} p_{2}-\frac{1}{2} \gamma \alpha+\frac{1}{2} \alpha+\frac{1}{4} \gamma p_{2}+\frac{1}{4} p_{3}-\frac{1}{4} \mu_{1}
$$

Solving for $\mu_{1}$ by substituting from (37) and (35)we have:

$$
\mu_{1}=2 \gamma \alpha-2 \alpha-3 \gamma p_{3}+p_{2}+\gamma p_{2}+p_{3}
$$


and substituting from (38) into (37)we derive $p_{1}$

$$
p_{1}=\gamma p_{3}-\alpha \gamma+\alpha
$$

this is the price reaction function for firm one when demand on its B market equal zero.

We then need to consider the case when demand, for firm one, is zero in the A market and positive for the B one, then $\mu_{1}=0$ and $\mu_{2}>0$

As before we solve for $\mu_{2}$ :

$$
\mu_{2}=2 \gamma \alpha-2 \alpha+\gamma p_{3}+p_{2}-3 \gamma p_{2}+p_{3}
$$

and by substituting (40) into (37)we obtain:

$$
p_{1}=-\alpha \gamma+\alpha+\gamma p_{2}
$$

the price reaction function when demand from $\mathrm{A}$, for firm one equals zero.

Finally when both demands are positive, no constraint is biting, the multipliers are both equal to zero $\mu_{1}=\mu_{2}=0$, and we have the internal reaction function so that:

$$
p_{1}=\frac{1}{4} \gamma p_{3}+\frac{1}{4} p_{2}-\frac{1}{2} \gamma \alpha+\frac{1}{2} \alpha+\frac{1}{4} \gamma p_{2}+\frac{1}{4} p_{3}
$$

Putting things together we obtain the reaction function for firm one:

$$
p_{1}=\left\{\begin{array}{ccc}
\gamma p_{2}-\gamma \alpha+\alpha & \text { if } & p_{2} \leq \frac{-\alpha+\alpha \gamma+p_{1}}{\gamma} \\
\frac{1}{4} \gamma p_{3}+\frac{1}{4} p_{2}-\frac{1}{2} \gamma \alpha+\frac{1}{2} \alpha+\frac{1}{4} \gamma p_{2}+\frac{1}{4} p_{3} & \text { if } & p_{2}>\frac{-\alpha+\alpha \gamma+p_{1}}{\gamma} \\
\gamma p_{3}-\alpha \gamma+\alpha & \text { if } & p_{3}>\frac{-\alpha+\alpha \gamma+p_{1}}{\gamma} \\
\hline \gamma & &
\end{array}\right.
$$

Firm two The Lagrangian for firm two is given by

$$
\begin{aligned}
L\left(p_{2}, \lambda\right)=p_{2}\left(\begin{array}{c}
\left(\frac{\alpha(1-\gamma)}{1-\gamma^{2}}-\frac{1}{1-\gamma^{2}} p_{3}+\frac{\gamma}{1-\gamma^{2}} p_{1}\right)+ \\
+\left(\frac{\alpha(1-\gamma)}{1-\gamma^{2}}-\frac{1}{1-\gamma^{2}} p_{1}+\frac{\gamma}{1-\gamma^{2}} p_{3}\right)+ \\
+\left(\frac{\alpha(1-\gamma)}{1-\gamma^{2}}-\frac{1}{1-\gamma^{2}} p_{2}+\frac{\gamma}{1-\gamma^{2}} p_{1}\right)
\end{array}\right)+ \\
+\lambda_{2}\left(\frac{\alpha(1-\gamma)}{1-\gamma^{2}}-\frac{1}{1-\gamma^{2}} p_{2}+\frac{\gamma}{1-\gamma^{2}} p_{1}\right)
\end{aligned}
$$


From the first order conditions we obtain :

$$
p_{2}=-\frac{3}{2} \gamma \alpha+\frac{1}{2} \gamma p_{3}+\gamma p_{1}+\frac{3}{2} \alpha-\frac{1}{2} p_{3}-\frac{1}{2} p_{1}-\frac{1}{2} \lambda_{2}
$$

When demand in $\mathrm{A}$ is zero, for firm two, we have: $\lambda_{2}>0$, and:

$$
\left(\frac{\alpha(1-\gamma)}{1-\gamma^{2}}-\frac{1}{1-\gamma^{2}} p_{2}+\frac{\gamma}{1-\gamma^{2}} p_{1}\right)=0
$$

solving for $\lambda_{2}$ we have:

$$
\lambda_{2}=\alpha-\gamma \alpha+\gamma p_{3}-p_{3}-p_{1}
$$

which again implies

$$
p_{2}=\gamma p_{1}-\gamma \alpha+\alpha
$$

When demand from $\mathrm{A}$ is positive $\lambda_{2}=0$ and (45) becomes

$$
p_{2}=-\frac{3}{2} \gamma \alpha+\frac{1}{2} \gamma p_{3}+\gamma p_{1}+\frac{3}{2} \alpha-\frac{1}{2} p_{3}-\frac{1}{2} p_{1}
$$

The reaction function for firm two is therefore:

$$
p_{2}=\left\{\begin{array}{ccc}
\gamma p_{1}-\gamma \alpha+\alpha & \text { if } & p_{1} \leq \frac{\gamma \alpha-\alpha+p_{2}}{\gamma} \\
-\frac{3}{2} \alpha \gamma+\frac{1}{2} \gamma p_{3}+\gamma p_{1}+\frac{3}{2} \alpha-\frac{1}{2} p_{3}-\frac{1}{2} p_{1} & \text { if } & p_{1}>\frac{\gamma \alpha-\alpha+p_{2}}{\gamma}
\end{array}\right.
$$

Firm three The Lagrangian is given by:

$$
\begin{aligned}
L_{3}= & \left(\frac{\alpha(1-\gamma)}{1-\gamma^{2}}-\frac{1}{1-\gamma^{2}} p_{3}+\frac{\gamma}{1-\gamma^{2}} p_{1}\right)\left(p_{3}-p_{2}\right)+ \\
& +\left(\frac{\alpha(1-\gamma)}{1-\gamma^{2}}-\frac{1}{1-\gamma^{2}} p_{1}+\frac{\gamma}{1-\gamma^{2}} p_{2}\right) p_{3}+ \\
& \varphi_{1}\left(\frac{\alpha(1-\gamma)}{1-\gamma^{2}}-\frac{1}{1-\gamma^{2}} p_{3}+\frac{\gamma}{1-\gamma^{2}} p_{1}\right)
\end{aligned}
$$

From the first order conditions we have:

$$
p_{3}=\frac{1}{2} \gamma p_{1}+\frac{1}{2} p_{2}-\gamma \alpha+\alpha+\frac{1}{2} \gamma p_{2}-\frac{1}{2} p_{1}-\frac{1}{2} \varphi_{1}
$$


When demand is zero in the $\mathrm{B}$ market then $\varphi_{1}>0$ and:

$$
\left(\frac{\alpha(1-\gamma)}{1-\gamma^{2}}-\frac{1}{1-\gamma^{2}} p_{3}+\frac{\gamma}{1-\gamma^{2}} p_{1}\right)=0
$$

Solving for $\varphi_{1}$ we have:

$$
\varphi_{1}=-(\gamma+1)\left(p_{1}-p_{2}\right)
$$

and substituting (54) into (52)we have the reaction function for firm two when demand from $\mathrm{B}$ is zero:

$$
p_{3}=\gamma p_{1}-\alpha \gamma+\alpha
$$

When demand in $\mathrm{B}$ is positive $\varphi_{1}=0$ and

$$
p_{3}=\frac{1}{2} \gamma p_{1}+\frac{1}{2} p_{2}-\frac{1}{2} \alpha \gamma+\frac{1}{2} \alpha+\frac{1}{2} \gamma p_{2}-\frac{1}{2} \alpha \gamma+\frac{1}{2} \alpha-\frac{1}{2} p_{1}
$$

firm three reaction function is therefore:

$$
p_{3}=\left\{\begin{array}{ccc}
\gamma p_{1}-\alpha \gamma+\alpha . & \text { if } & p_{1} \leq \frac{\alpha \gamma-\alpha+p_{3}}{\gamma} \\
\frac{1}{2} \gamma p_{1}+\frac{1}{2} p_{2}-\alpha \gamma+\alpha+\frac{1}{2} \gamma p_{2}-\frac{1}{2} p_{1} & \text { if } & p_{1}>\frac{\alpha \gamma-\alpha+p_{3}}{\gamma}
\end{array}\right.
$$

Internal solutions are equilibrium solutions

From (43), (50) and (57)the system of the internal reaction functions is given by:

$$
\begin{aligned}
& p_{3}=\frac{1}{2} \gamma p_{1}+\frac{1}{2} p_{2}-\alpha \gamma+\alpha+\frac{1}{2} \gamma p_{2}-\frac{1}{2} p_{1} \\
& p_{2}=-\frac{3}{2} \alpha \gamma+\frac{1}{2} \gamma p_{3}+\gamma p_{1}+\frac{3}{2} \alpha-\frac{1}{2} p_{3}-\frac{1}{2} p_{1} \\
& p_{1}=\frac{1}{4} \gamma p_{3}+\frac{1}{4} p_{2}-\frac{1}{2} \alpha \gamma+\frac{1}{2} \alpha+\frac{1}{4} \gamma p_{2}+\frac{1}{4} p_{3}
\end{aligned}
$$

solved by:

$$
\begin{aligned}
& p_{1}=\alpha \frac{4 \gamma-7+3 \gamma^{2}}{3 \gamma^{2}+3 \gamma-8} \\
& p_{2}=\left(3 \gamma^{2}+2 \gamma-5\right) \frac{\alpha}{3 \gamma^{2}+3 \gamma-8} \\
& p_{3}=\alpha \frac{4 \gamma-7+3 \gamma^{2}}{3 \gamma^{2}+3 \gamma-8}
\end{aligned}
$$


To verify that (59) forms an equilibrium for the game we need to check if they satisfy the constraints in the reaction function system.

From (43) we want:

$$
p_{2}>\frac{-\alpha+\alpha \gamma+p_{1}}{\gamma}
$$

and

$$
p_{3}>\frac{-\alpha+\alpha \gamma+p_{1}}{\gamma}
$$

and indeed in equilibrium (60) is verified since:

$$
\left(3 \gamma^{2}+2 \gamma-5\right) \frac{\alpha}{3 \gamma^{2}+3 \gamma-8}>\alpha \frac{3 \gamma^{3}+3 \gamma^{2}-7 \gamma+1}{\gamma\left(3 \gamma^{2}+3 \gamma-8\right)}
$$

implies that

$$
-\alpha \frac{\gamma^{2}-2 \gamma+1}{\gamma\left(3 \gamma^{2}+3 \gamma-8\right)}>0
$$

and this is verified for $\gamma<1$.

The second condition (61) implies:

$$
\alpha \frac{4 \gamma-7+3 \gamma^{2}}{3 \gamma^{2}+3 \gamma-8}>\alpha \frac{3 \gamma^{3}+3 \gamma^{2}-7 \gamma+1}{\gamma\left(3 \gamma^{2}+3 \gamma-8\right)}
$$

or

$$
\alpha \frac{\gamma^{2}-1}{\gamma\left(3 \gamma^{2}+3 \gamma-8\right)}>0
$$

and this is also verified for $\gamma<1$.

For firm two to play the internal solution, from (50), we want:

$$
p_{1}>\frac{\gamma \alpha-\alpha+p_{2}}{\gamma}
$$

This verified in the internal equilibrium, in facts:

$$
\alpha \frac{4 \gamma-7+3 \gamma^{2}}{3 \gamma^{2}+3 \gamma-8}>3 \alpha \frac{\gamma^{3}+\gamma^{2}-3 \gamma+1}{\gamma\left(3 \gamma^{2}+3 \gamma-8\right)}
$$

or

$$
\alpha \frac{\gamma^{2}+2 \gamma-3}{\gamma\left(3 \gamma^{2}+3 \gamma-8\right)}>0
$$

which is again always verified for $\gamma<1$. 
For firm three to play internal, from (57), we want

$$
p_{1}>\frac{\alpha \gamma-\alpha+p_{3}}{\gamma}
$$

For (69) to hold in equilibrium we need:

$$
\alpha \frac{4 \gamma-7+3 \gamma^{2}}{3 \gamma^{2}+3 \gamma-8}-\alpha \frac{3 \gamma^{3}+3 \gamma^{2}-7 \gamma+1}{\gamma\left(3 \gamma^{2}+3 \gamma-8\right)}>0
$$

or

$$
\alpha \frac{\gamma^{2}-1}{\gamma\left(3 \gamma^{2}+3 \gamma-8\right)}>0
$$

but this is always the case when $\gamma<1$.

Therefore (59) is the internal equilibrium for the system of reaction functions: (43), (50) and (57). 


\section{References}

[1] Armstrong, M.(1998). "Network Interconnection in Telecommunications", The Economic Journal, vol 108, pp. 545-564.

[2] Armstrong, M., Doyle, C. and Vickers, J. (1996) "The Access Pricing Problem: a synthesis", Journal of Industrial Economics, vol.44 pp 131150.

[3] Danish Internet Exchange Website: http://www.unic.dk/dix*/euro.html

[4] Economides, N. (1998), "The Telecommunications Act of 1996 and its Impact," Discussion Paper EC-98-08, Stern School of Business, N.Y.U., forthcoming, Japan and the World Economy.

[5] Economides, N. (1998), "The Incentive for non-price discrimination by an input monopolist". International Journal of Industrial Organization, vol.16, pp. 271-284.

[6] Economides, N., and Woroch, G., (1992), "Benefits and Pitfalls of Network Interconnection," Discussion Paper no. EC-92-31, Stern School of Business.

[7] A. Estache, and Valletti, T. (1999),"The theory of access pricing: an overview for infrastructure regulators", Discussion Paper 2133, CEPR, and Policy Research Working Paper 2097, The World Bank.

[8] Gibbens, R. at http://www.statslab.cam.ac.uk/ richard/PRICE/pricinginternet/

[9] Hobbes, R. Zakon "Hobbes' Internet Timeline v4.", http://www.isoc.org/guest/zakon/Internet/History/HIT.html

[10] Laffont, J.-J., Rey, P. and Tirole, J. (1998) Network Competition: I.Overview and nondiscriminatory pricing", Rand Journal of Economics, Vol 29, 1. pp 1-37.

[11] MacKie-Mason and H. R. Varian,(1995) "Pricing the Internet," in Public Access to the Internet, B. Kahin and J. Keller, eds., MIT Press, 1995, pp. 269-314. 
[12] Shenker, S., D. Clark, D. Estrin, and S. Herzog, (1996); "Pricing in computer networks: reshaping the research agenda," Telecommunications Policy, 20, pp.183-201.

[13] Valletti, T. and Cambrini, C., "Il Mercato di Internet: aspetti economici e problemi di tariffazione" mimeo. 